\title{
Natural Products, Traditional Uses and Pharmacological Activities of the Genus Biebersteinia (Biebersteiniaceae)
}

\author{
Benyin Zhang ${ }^{1,2, *,+}+\mathbb{C}$, Xiaona Jin ${ }^{3,+}$, Hengxia Yin ${ }^{1}$, Dejun Zhang ${ }^{1,2}$, Huakun Zhou ${ }^{4}$, \\ Xiaofeng Zhang ${ }^{3}$ and Lam-Son Phan Tran ${ }^{5,6, * \mathbb{D}}$ \\ 1 State Key Laboratory of Plateau Ecology and Agriculture, Qinghai University, Xining 810016, China; \\ hengxiayin@qhu.edu.cn (H.Y.); djzhang@nwipb.cas.cn (D.Z.) \\ 2 Laboratory of Natural Product Research, College of Eco-Environmental Engineering, Qinghai University, \\ Xining 810016, China \\ 3 Institute of Life Science Application, College of Medicine, Xi'an International University, \\ Xi'an 710017, China; xaiu05029@xaiu.edu.cn (X.J.); xaiu16041@xaiu.edu.cn (X.Z.) \\ 4 The Key Laboratory of Restoration Ecology in Cold Region of Qinghai Province, Northwest Institute of \\ Plateau Biology, Chinese Academy of Science, Xining 810008, China; hkzhou@nwipb.cas.cn \\ 5 Institute of Research and Development, Duy Tan University, 03 Quang Trung, Da Nang 550000, Vietnam \\ 6 Stress Adaptation Research Unit, RIKEN Center for Sustainable Resource Science, 1-7-22, Suehiro-cho, \\ Tsurumi, Yokohama 230-0045, Japan \\ * Correspondence: benyinzhang@qhu.edu.cn (B.Z.); tranplamson@duytan.edu.vn or \\ son.tran@riken.jp (L.-S.P.T.) \\ + These authors contributed equally to this work.
}

Received: 26 March 2020; Accepted: 28 April 2020; Published: 7 May 2020

\begin{abstract}
Medicinal plants have been known as a rich source of natural products (NPs). Due to their diverse chemical structures and remarkable pharmacological activities, NPs are regarded as important repertoires for drug discovery and development. Biebersteinia plant species belong to the Biebersteiniaceae family, and have been used in folk medicines in China and Iran for ages. However, the chemical properties, bioactivities and modes of action of the NPs produced by medicinal Biebersteinia species are poorly understood despite the fact that there are only four known Biebersteinia species worldwide. Here, we reviewed the chemical classifications and diversity of the various NPs found in the four known Biebersteinia species. We found that the major chemical categories in these plants include flavonoids, alkaloids, phenylpropanoids, terpenoids, essential oils and fatty acids. We also discussed the anti-inflammatory, analgesic, antibacterial, antioxidant, antihypertensive and hypoglycemic effects of the four Biebersteinia species. We believe that the present review will facilitate the exploration of traditional uses and pharmacological properties of Biebersteinia species, extraction of the NPs and elucidation of their molecular mechanisms, as well as the development of novel drugs based on the reported properties and mode-of-action.
\end{abstract}

Keywords: Biebersteinia; Biebersteiniaceae; chemical properties; natural products; traditional uses; pharmacological activities

\section{Introduction}

Biebersteinia is the smallest genus of Biebersteiniaceae. The systemic and taxonomic position of this genus has long been in dispute due to its rare species and limited availability of representative herbarium collections [1]. The genus was traditionally positioned in Geraniales 30 years ago, but now it was accepted that it belongs to Sapindales as a separate order and family based on the molecular 
phylogenetic analysis [2,3]. The genus Biebersteinia was originally recognized to comprise five species-namely, B. heterostemon Maxim., B. multifida DC., B. leiosepala Jaub. \& Spach, B. odora Stephan ex Fisch. and B. orphanidis Boiss. decades ago [1]. However, B. leiosepala is now recognized to be a synonymous species of B. multifida (http://www.theplantlist.org/, http://www.worldfloraonline.org/, and https://www.gbif.org/); and thus, there are a total of four species in the genus. These species are widely distributed in mountainous, semi-arid regions from central and western Asia to the eastern Mediterranean [1,2,4-6].

All of the Biebersteinia species are perennial herbs, and possess slightly different biological characteristics and geographical distributions. B. heterostemon, also called "Xun Dao Niu" in Chinese, is endemic to the Qinghai-Tibetan Plateau and its adjacent regions in China [6]. This plant species inhabits arid and semi-arid alpine deserts, rocky slopes and other environments (http://www.iplant.cn/foc/). The morphological and biological characteristics of B. heterostemon are $40-120 \mathrm{~cm}$ tall, lanceolate leaf blade bearing long simple hairs and small stipitate glands, flowers in two or three fascicles with hairy or glandular pedicel, as well as yellow and obovate petals (http://www.iplant.cn/foc/). B. multifida is a common herb known as Adamak in Iran, with $20-70 \mathrm{~cm}$ long stem, laciniate leaves, flowers formed in a lax panicle, calyx strengthened in fruit, and yellowish petals slightly shorter than the sepals [7]. B. odora is distributed widely across central Asia (e.g., Kazakhstan, Kyrgyzstan, Pakistan, India, China and Mongolia) and inhabited in alpine meadows and dry rocky and scree slopes. B. odora is $10-30 \mathrm{~cm}$ tall, and has pinnatisect leaves and yellow flowers with orange center $(1-1.5 \mathrm{~cm}$ across, in racemes) [8]. B. orphanidis is the only species distributed in Europe and found in Greece besides Asia. B. orphanidis grows at altitudes $\sim 1400-1750 \mathrm{~m}$ in deep sandy-clay soil in dolines over limestone, usually in openings of Abies cephalonica forest. These plants are $15-40 \mathrm{~cm}$ long, broadly oblanceolate in outline, with scarious stipules and short petioles [5].

Of these species, B. heterostemon and B. multifida have long histories as traditional folk medicines in Iran and the Tibetan region of China, respectively, and have been used to treat various diseases. Modern pharmacological studies have shown that these two plant species have significant pharmaceutical effects on humans, and possess various ethnomedicinal properties, including antioxidant, analgesic, anti-inflammatory, antispasmodic, hypoglycemic, hypotensive and anti-atherosclerotic properties [9-11]. Therefore, numerous phytochemists and pharmacologists worldwide have investigated the pharmacodynamically active substances in Biebersteinia species. Natural products isolated from Biebersteinia plants include flavonoids, guanidines, alkaloids, phenylpropanoids, terpenoids, sterols and fatty acids, as well as various compounds of essential oils. The present review summarizes the findings of several decades of research into the chemical constituents and pharmacological functions of the four identified Biebersteinia species. This review, therefore, will facilitate further investigations into the complete chemical profile of the secondary metabolites in these plants, as well as their pharmacological activities and molecular mechanisms.

\section{Data Collections}

All data presented in this review were summarized from the references, including scientific journals, book chapters or dissertations. These references were systematically searched against electronic databases: PubMed, CNKI (http://new.oversea.cnki.net/index/), Web of Science, Scopus and Google Scholar with a keyword "Biebersteinia". To search for maximum relative references, the keyword was set as "Biebersteinia" without any other restrictions. Subsequently, references closely related to chemical compositions, traditional uses and pharmacological properties, including in vitro and in vivo investigations, were screened for further data extraction. In addition, to survey the taxon, phenotypes and geographical distributions of species in Biebersteinia, several online taxonomic databases, including http://theplantlist.org/, http://www.worldfloraonline.org/, https://www.gbif.org/ and http://www.iplant.cn/foc/, were also explored. 


\section{Natural Products Isolated from Bieberstrinia}

\subsection{Flavonoids}

Up-to-date, 29 flavonoids (Figures 1 and 2; Table 1) have been isolated from four Biebersteinia species, which occupies most of the known chemicals in Biebersteinia species. The flavonoid aglycones comprise mainly flavones or flavonols, such as quercetin, luteolin and apigenin. Fifteen aglycone derivatives have been discovered, among which 12 compounds are flavones (1-12) and three are flavonols (13-15) (Figure 1; Table 1). Fourteen flavonoid glycosides with different types or numbers of glycosyl moiety, including 11 flavone (16-26) and three flavonol glycosides (27-29) were found (Figure 2; Table 1).

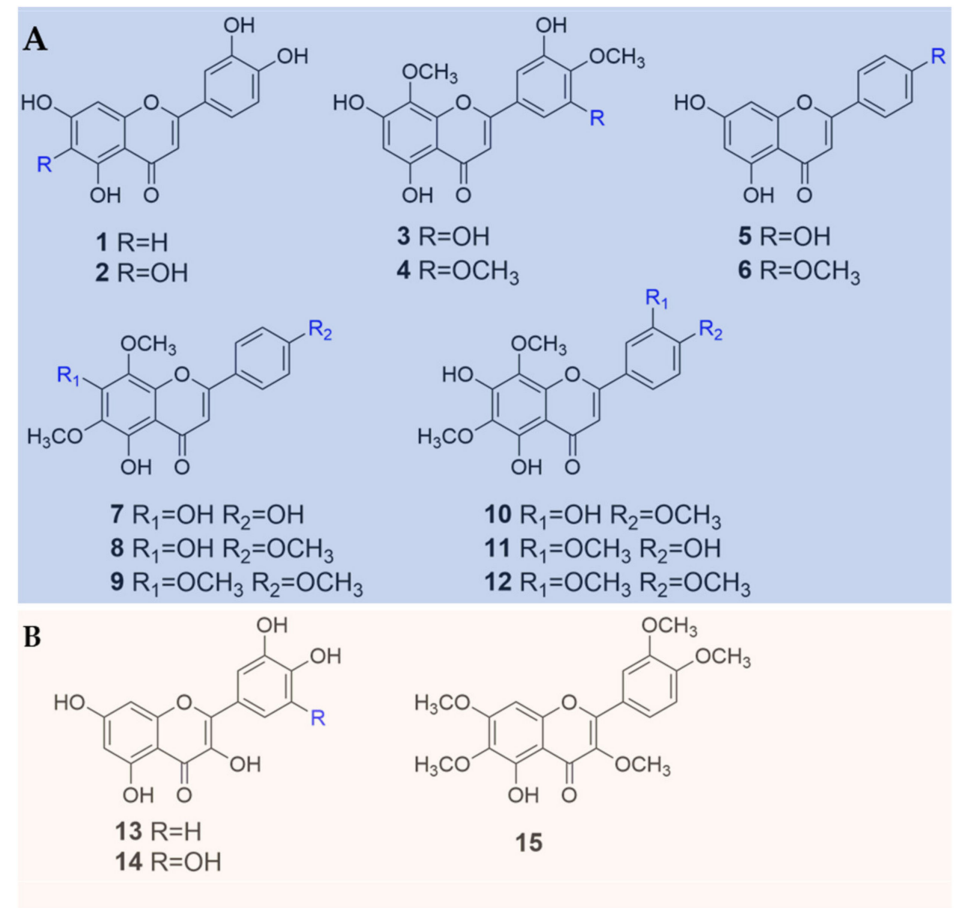

Figure 1. Chemical structures of 15 flavonoid aglycones identified in Biebersteinia plants. (A) flavone aglycones; (B) flavonol aglycones.

In addition, most of the flavonoid aglycones and glycosides were highly hydroxy- or methoxy-substituted at C-6, C-8, C-3', C-4' and C-5' in their chemical structures (Figures 1 and 2). Both C-6 and C-8 were substituted by methoxy groups as seen in the chemical structures of compounds 7-12 (Figure 1). To the best of our knowledge, this configuration occurs rarely in nature, which might be correlated with their extreme habitats. From the sources of flavonoids, 18 compounds were isolated from the species $B$. heterostemon (1-4, 13, 15, 16-19 and 22-29) [11-14], among which compounds 3 and 18 were recently discovered by our group from the species for the first time [14]. Compounds 1, 19, 23, 5-12 and 20-21 were mainly identified from B. orphanidis [15], while three flavonoids (1, $\mathbf{7}$ and 12) were found in B. multifida; however, only one compound, namely myricetin (14), was reported from B. odora [16] (Table 1).

The content of total flavonoids (CTF) in plants may be correlated with their habitats, ecological roles and responses to abiotic and/or biotic stresses [17-19]. In general, the Biebersteinia species are widely distributed at high elevations, and are exposed to extreme drought, low temperatures and strong ultraviolet radiation [20]. All of these conditions could induce high production of CTF. One of our previous studies showed that the CTF reached $0.24 \%$ in B. heterostemon located on the Qinghai-Tibetan Plateau [21]. CTF may also widely vary among different plant organs and tissues. For instance, 
in B. multifida, leaves were found to have the highest CTF $(39.9 \pm 2.1 \mathrm{mg} / \mathrm{g})$, followed by flowers, stems and roots [22].

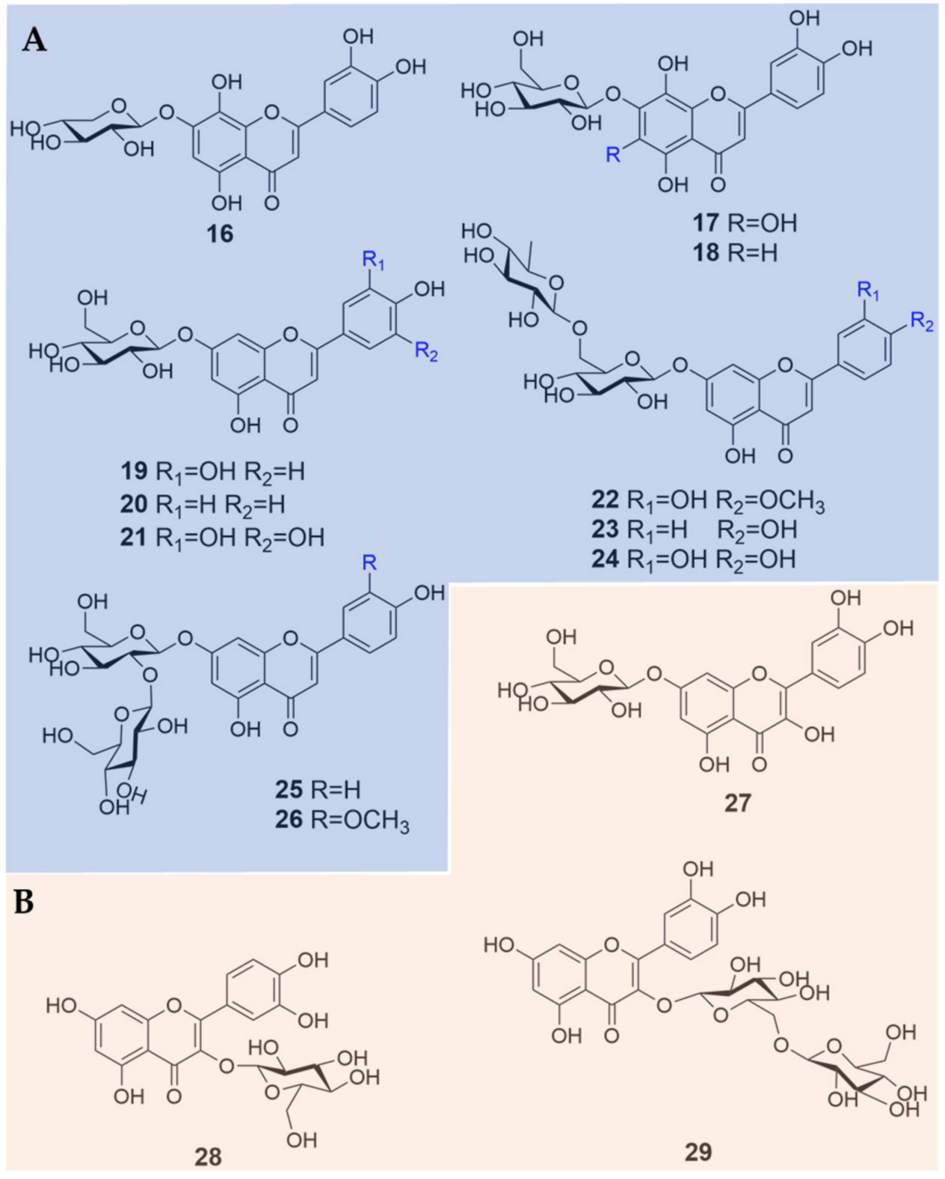

Figure 2. Chemical structures of 14 flavonoid glycosides identified in Biebersteinia plants. (A) flavone glycosides; (B) flavonol glycosides.

Table 1. Chemical constituents (except essential oil-related compounds) in Biebersteinia species.

\begin{tabular}{|c|c|c|c|}
\hline No. & Compound Name & Sources & References \\
\hline \multicolumn{4}{|c|}{ Flavonoids } \\
\hline 1 & luteolin & $\begin{array}{c}\text { B. heterostemon } \\
\text { B. multifida } \\
\text { B. orphanidis }\end{array}$ & {$[11,13,15]$} \\
\hline 2 & 6-hydroxyluteolin & B. heterostemon & [13] \\
\hline 3 & $4^{\prime}$-methoxytricetin & B. heterostemon & [14] \\
\hline 4 & $5,7,3^{\prime}$-trihydroxy- $8,4^{\prime}, 5^{\prime}$-trimethoxyflavone & B. heterostemon & [11] \\
\hline 5 & apigenin & B. orphanidis & [15] \\
\hline 6 & acacetin & B. orphanidis & [15] \\
\hline 7 & 5,7,4'-trihydroxy-6,8-dimethoxyflavone & B. orphanidis & [15] \\
\hline 8 & nevadensin & B. orphanidis & {$[15]$} \\
\hline 9 & gardenin B & B. orphanidis & [15] \\
\hline 10 & acerosin & B. orphanidis & [15] \\
\hline 11 & sudachitin & B. orphanidis & [15] \\
\hline 12 & hymenoxin & B. orphanidis & [15] \\
\hline 13 & quercetin & B. heterostemon & [12] \\
\hline 14 & myricetin & B. odora & [16] \\
\hline 15 & artemetin & B. heterostemon & [23] \\
\hline 16 & hypolaetin-7-O- $\beta$-D-xylopyranoside & B. heterostemon & [12] \\
\hline 17 & hypolaetin-7-O- $\beta$-D-glucopyranoside & B. heterostemon & [13] \\
\hline 18 & $3^{\prime}, 4^{\prime}, 5,8$-tetrahydroxyflavanone-7-O- $\beta$-glucopyranoside & B. heterostemon & [24] \\
\hline
\end{tabular}


Table 1. Cont

\begin{tabular}{|c|c|c|c|}
\hline No. & Compound Name & Sources & References \\
\hline 19 & luteolin-7-O-glucoside & $\begin{array}{l}\text { B. heterostemon } \\
\text { B. multifida } \\
\text { B. orphanidis }\end{array}$ & {$[11,13,15]$} \\
\hline 20 & apigenin-7-O-glucoside & B. orphanidis & [15] \\
\hline 21 & tricetin-7-O-glucoside & B. orphanidis & [15] \\
\hline 22 & diosmin & B. heterostemon & [13] \\
\hline 23 & apigenin-7-O-rutinoside & $\begin{array}{l}\text { B. heterostemon } \\
\text { B. orphanidis }\end{array}$ & {$[13,15]$} \\
\hline 24 & luteolin-7-O-rutinoside & $\begin{array}{l}\text { B. heterostemon } \\
\text { B. multifida }\end{array}$ & {$[13,15]$} \\
\hline 25 & apigenin-7-O-sophoroside & B. heterostemon & [13] \\
\hline 26 & chrysoeriol-7-O-sophoroside & B. heterostemon & [13] \\
\hline 27 & quercetin-7-O-glucoside & B. heterostemon & [11] \\
\hline 28 & quercetin-3-O- $\beta$-glucopyranoside & B. heterostemon & [13] \\
\hline 29 & $\begin{array}{l}\text { quercetin-3-O- } \beta \text {-D-glucopyranosyl } \\
\quad(1 \rightarrow 2)-\beta \text {-D-glucopyranoside }\end{array}$ & B. heterostemon & [13] \\
\hline \multicolumn{4}{|c|}{ Guanidines } \\
\hline 30 & galegine & B. heterostemon & {$[12,25]$} \\
\hline 31 & cis-4-hydroxy galegine & B. heterostemon & [25] \\
\hline 32 & trans-4-hydroxy galegine & B. heterostemon & [25] \\
\hline \multicolumn{4}{|c|}{ Phenylpropanoids } \\
\hline 33 & umbelliferone & B. multifida & [23] \\
\hline 34 & scopoletin & B. multifida & [23] \\
\hline 35 & ferulic acid & B. multifida & [23] \\
\hline \multicolumn{4}{|c|}{ Terpenoids } \\
\hline 36 & geniposide & B. heterostemon & [26] \\
\hline 37 & $6 \beta$-hydroxy geniposide & B. heterostemon & [26] \\
\hline \multicolumn{4}{|c|}{ Other Types } \\
\hline 39 & (+)-dehydrovomifoliol & B. heterostemon & [24] \\
\hline 40 & $N$-3-methyl-2-butenyl urea & B. heterostemon & [11] \\
\hline 41 & vasicinone & B. multifida & [27] \\
\hline 42 & alternariol & B. heterostemon & [24] \\
\hline 43 & mannitol & B. heterostemon & [12] \\
\hline 44 & $\beta$-sitosterol & B. heterostemon & {$[11,12,24]$} \\
\hline 45 & daucosterol & B. heterostemon & [12] \\
\hline \multicolumn{4}{|c|}{ Fatty Acids } \\
\hline 47 & myristic acid & B. orphanidis & [28] \\
\hline 48 & palmitic acid & $\begin{array}{l}\text { B. heterostemon } \\
\text { B. orphanidis }\end{array}$ & {$[28,29]$} \\
\hline 49 & stearic acid & $\begin{array}{l}\text { B. heterostemon } \\
\text { B. orphanidis }\end{array}$ & {$[28,29]$} \\
\hline 50 & arachidic acid & B. heterostemon & [29] \\
\hline 51 & docosanoic acid & B. orphanidis & [28] \\
\hline 52 & tetracosanoic acid & B. orphanidis & [28] \\
\hline 53 & hexacosanoic acid & B. orphanidis & [28] \\
\hline 54 & palmitoleic acid & $\begin{array}{l}\text { B. heterostemon } \\
\text { B. orphanidis }\end{array}$ & {$[28,29]$} \\
\hline 55 & oleic acid & $\begin{array}{l}\text { B. heterostemon } \\
\text { B. orphanidis }\end{array}$ & {$[28,29]$} \\
\hline 56 & eicosenoic acid & $\begin{array}{l}\text { B. heterostemon } \\
\text { B. orphanidis }\end{array}$ & {$[28,29]$} \\
\hline 57 & linoleic acid & $\begin{array}{l}\text { B. heterostemon } \\
\text { B. orphanidis }\end{array}$ & {$[28,29]$} \\
\hline 58 & $\alpha$-linolenic acid & $\begin{array}{l}\text { B. heterostemon } \\
\text { B. orphanidis }\end{array}$ & {$[28,29]$} \\
\hline 59 & $\gamma$-linolenic acid & B. heterostemon & [29] \\
\hline 60 & 7,10,13-hexadecatrienoic acid & B. orphanidis & [28] \\
\hline
\end{tabular}




\subsection{Guanidines}

Three rare prenylated guanidines, namely galegine (30), cis-4-hydroxy galegine (31) and trans-4-hydroxy galegine (32) [30,31], have been found in B. heterostemon [25] (Figure 3A; Table 1). The clinical hypoglycemic drug metformin was derived from galegine, which might account for the hypoglycemic efficacy of the traditional galegine-containing Tibetan medicine B. heterostemon. In fact, numerous alkaloids [14], such as coptisonine [32], conophylline [33] and vindogentianine [34], can induce hypoglycemia.

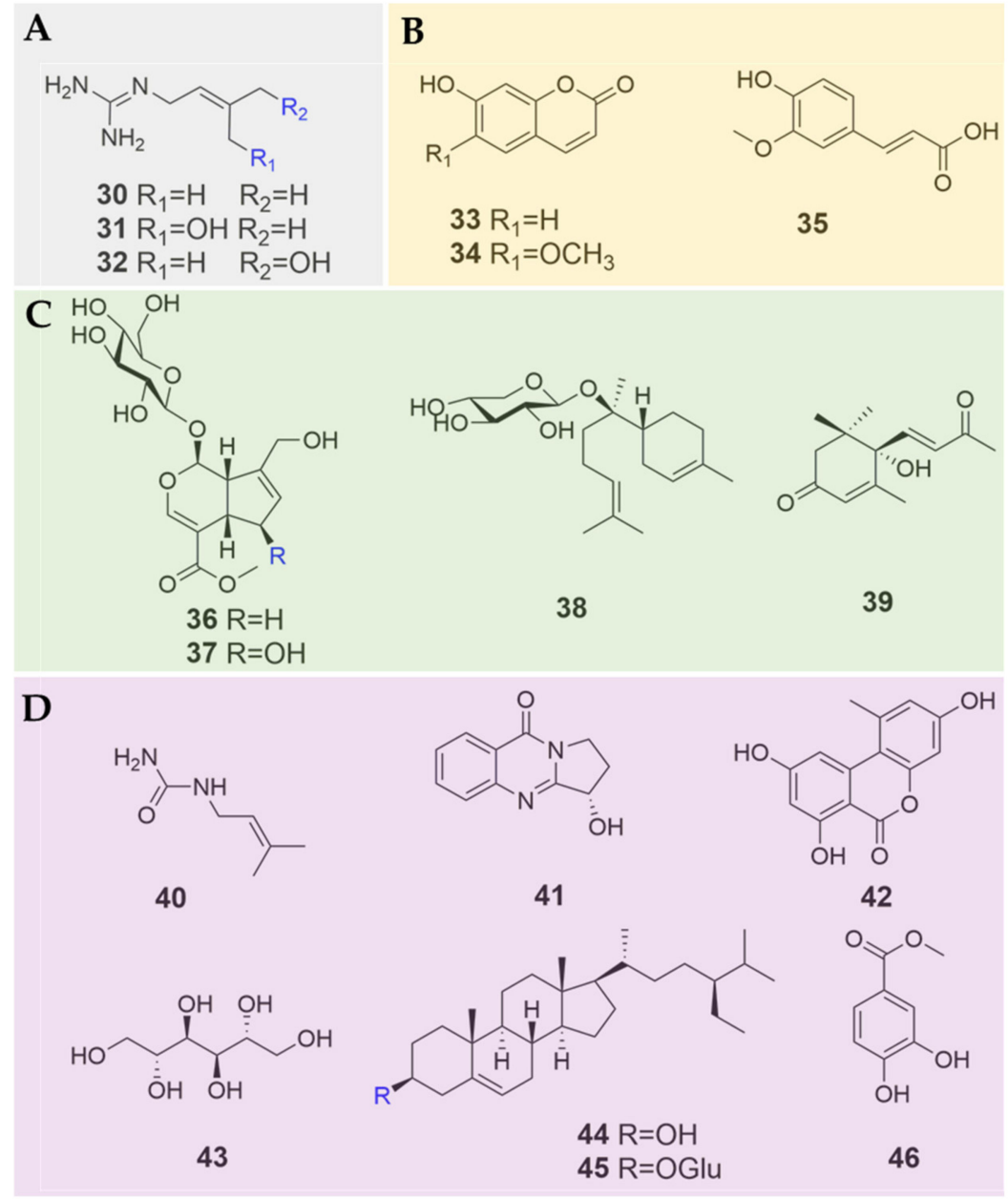

Figure 3. Chemical structures of guanidines (A) phenylpropanoids, (B) terpenoids, (C) and other compounds, (D) identified in Biebersteinia species.

\subsection{Phenylpropanoids}

Three phenylpropanoids have been isolated so far from two Biebersteinia species (Figure 3B; Table 1). Compounds $\mathbf{3 3}$ and $\mathbf{3 4}$ are coumarins that were identified in B. multifida, which differ only in terms of their substituents at C-6. Compound 35 is a ferulic acid that was isolated from B. multifida [23], as well as from B. heterostemon by our group (unpublished data).

\subsection{Terpenoids}

Until the present, four terpenoids have been isolated from $B$. heterostemon among the four Biebersteinia species. These identified terpenoids include two iridoid glucosides, i.e., geniposide (36) and $6 \beta$-hydroxygeniposide (37), and one sesquiterpene glycoside (-)-anymol-8-O- $\beta$-D-lyxopyranoside (38) [26]. They are the main active ingredients [35], and are easily hydrolyzed by $\beta$-glucosidase to genipin [36]. In addition, we recently isolated one sesquiterpene (+)-dehydrovomifoliol (39) from 
B. heterostemon, the identification and characterization of which were also reported for the first time from the genus Biebersteinia (Figure 3C; Table 1).

\subsection{Other Compounds}

Seven other types of compounds were isolated from $B$. heterostemon, including N-3-methyl-2-butenylurea (40) [11], alkaloid vasicinone (41) [27], alternariol (42) [24], mannitol (43) [12], $\beta$-sitosterol (44) [11,12,24], daucosterol (45) [12], and protocatechuic acid methyl ester (46) [24] (Figure 3D; Table 1). In addition, three neutral polysaccharides were obtained from the roots of B. multifida, namely, glucan-A, glucan-B and glucan-C. Their molecular weights were 4100, 2200 and 1100 , respectively [37-40].

\subsection{Fatty Acids}

Fatty acids, which are aliphatic monocarboxylic acids, can either be saturated or unsaturated depending on the absence or presence of double bonds [41]. A number of studies have reported the presence of various fatty acids in two out of four Biebersteinia species [28,29]. In particular, a total of 14 fatty acids were identified in the seed oil of B. heterostemon and leaves of B. orphanidis (Figure 4; Table 1). These fatty acids include seven saturated fatty acids (47-53), three monounsaturated fatty acids (54-56), and four polyunsaturated fatty acids (57-60). By using gas chromatography (GC) analysis of fatty acids in seed oil of B. heterostemon, nine fatty acids (48-50 and 54-59) were identified, which accounted for $88.44 \%$ of total fatty acid content that mainly consisted of unsaturated fatty acids, such as oleic (55), linoleic (57) and linolenic (58 and 59) acids, while the lower detected part (7.94\%) of total fatty acid content contained saturated fatty acids, mainly palmitic (48) and stearic (49) acids [29]. In particular, the content of linoleic acid reached $73.04 \%$ [29]. Twelve fatty acids were elucidated in the leaves of B. orphanidis (47-49, 51-58 and 60), among which palmitic, linolenic and linoleic acids were predominant, representing $30.60 \%, 21.83 \%$ and $11.67 \%$, respectively, in total fatty acid content [28].

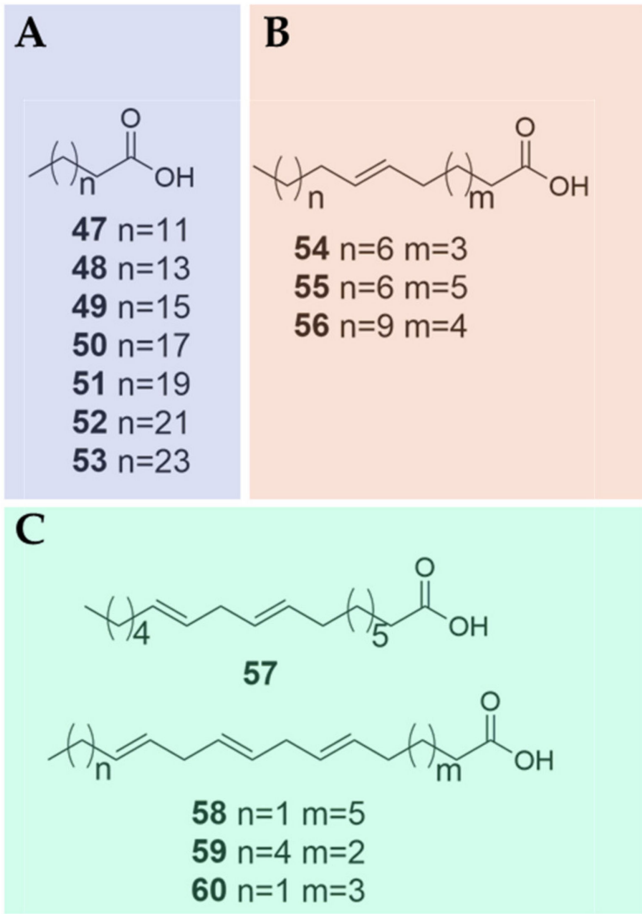

Figure 4. Chemical structures of fatty acids identified in Biebersteinia species. (A) saturated fatty acids; (B) monounsaturated fatty acids; (C) polyunsaturated fatty acids. 


\section{Chemical Compositions of Essential Oils in Biebersteinia Species}

Up-to-date, 112 chemical constituents have been identified in the essential oils of three Biebersteinia species, namely B. multifida, B. heterostemon and B. orphanidis [42-48], mainly by using gas chromatography-mass spectrometry (GC-MS) analyses (Table 2). In particular, the chemical compositions of essential oils of $B$. multifida were more systematically investigated, using different types of tissues, such as leaves, fruits and roots [45], or using different extraction methods, such as hydrodistillation, microwave, solvent and supercritical fluid extraction (SFE) [42,44]. In the essential oil of B. multifida, a total of 88 chemical constituents were identified [45-48]. The chemodiversity and contents of various compounds in essential oils from different parts of B. multifida differed significantly [45]. Specifically, thymol (16.5\% of total essential oil), $\alpha$-pinene (14.3\%), $\beta$-pinene $(12.4 \%), \beta$-caryophyllene $(11.2 \%)$ and 1,8 -cineol $(10.1 \%)$ are the major compounds in essential oil extracted from B. multifida leaves; thymol (38.4\%), 1,8-cineol $(18.4 \%), \gamma$-terpinene $(11.3 \%)$ and $\beta$-caryophyllene $(9.8 \%)$ are the main compounds in essential oil extracted from B. multifida roots; and thymol $(30.9 \%), \beta$-caryophyllene $(15.5 \%), \alpha$-pinene $(9.4 \%), \beta$-pinene $(8.8 \%)$, caryophyllene oxide $(8.4 \%)$ and limonene $(7.5 \%)$ are predominant in essential oil extracted from $B$. multifida fruits [45]. Additionally, different extraction methods were also shown to induce different chemical types or contents in essential oil extracts of B. multifida. For example, the hydrodistillation method enabled the authors to mainly detect $(E)$-nerolidol (31.45\%) and phytol (17.1\%); microwave extraction allowed detection of $(E)$-nerolidol $(28.4 \%), n$-heptacosane $(17.36 \%), n$-docosane $(12.97 \%)$ and 6,10,14-trimethyl-2-pentadecanone (10.38\%); while solvent extraction detected mainly nonacosane (38.62\%), mandenol (17.17\%) and $n$-heptacosane (10.23\%) [44]. In addition to the above-mentioned extraction approaches of essential oils, the supercritical fluid extraction (SFE) is a green technology that has been widely used in the past few decades to extract essential oils, nonpolar substances, fatty acids, phytosterols, and other functional and nutraceutical components from natural sources [49-54]. Four compounds, namely nerolidol, 6,10,14-trimethyl-2-pentadecanone, hexadecanoic acid and phytol that possess strong antioxidant activities, were the major components in essential oils extracted from aerial parts of B. multifida by both hydrodistillation and SFE methods; however, the yield of these four compounds extracted by SFE (91.74\%) was far higher than that by the hydrodistillation method [42].

In comparison with $B$. multifida, the investigations of chemical compositions of essential oils extracted from B. heterostemon and B. orphanidis were still limited. Forty compounds $(82.43 \%$ of total essential oil) were identified in the essential oil of aerial parts of $B$. heterostemon, mainly containing $\beta$-caryophyllene (33.79\%), elixene (5.09\%), $\beta$-elemenone $(4.45 \%)$, germacrene $\mathrm{D}(3.64 \%)$, camphor (3.34\%), $\alpha$-bisabolol (3.30\%) and geraniol (3.27\%) [48]. Thirteen components constituting $98.12 \%$ of the essential oil extracted from the aerial parts of B. orphanidis were detected, and the major chemical constituents included cis-limonene oxide (47.90\%), $\beta$-caryophyllene $(9.70 \%)$ and $\alpha$-bisabolol $(8.23 \%)$ [43]. Furthermore, oxygenated monoterpenes $(51.25 \%)$ were found to be predominated over other chemical types in the constituent composition of the B. orphanidis essential oil in this study [43].

Table 2. Chemical compositions of essential oils in Biebersteinia species.

\begin{tabular}{|c|c|c|c|c|c|}
\hline No. & Compound Name & Molecular Formula & Retention Indices (RI) & Sources & References \\
\hline 1 & $\alpha$-thujene & $\mathrm{C}_{10} \mathrm{H}_{16}$ & 920 & B. multifida & [47] \\
\hline 2 & $\alpha$-pinene & $\mathrm{C}_{10} \mathrm{H}_{16}$ & 939 & $\begin{array}{l}\text { B. multifida } \\
\text { B. heterostemon }\end{array}$ & {$[44-46,48]$} \\
\hline 3 & camphene & $\mathrm{C}_{10} \mathrm{H}_{16}$ & 946 & $\begin{array}{l}\text { B. multifida } \\
\text { B. heterostemon }\end{array}$ & {$[45,48]$} \\
\hline 4 & sabinene & $\mathrm{C}_{10} \mathrm{H}_{16}$ & 970 & B. multifida & [45] \\
\hline 5 & $\beta$-pinene & $\mathrm{C}_{10} \mathrm{H}_{16}$ & 978 & $\begin{array}{l}\text { B. multifida } \\
\text { B. heterostemon }\end{array}$ & {$[45,48]$} \\
\hline 6 & 6-methyl-5-hepten-2-one & $\mathrm{C}_{8} \mathrm{H}_{14} \mathrm{O}$ & 988 & B. multifida & [47] \\
\hline 7 & myrcene & $\mathrm{C}_{10} \mathrm{H}_{16}$ & 991 & $\begin{array}{l}\text { B. multifida } \\
\text { B. heterostemon }\end{array}$ & {$[45,48]$} \\
\hline 8 & $\alpha$-phellandrene & $\mathrm{C}_{10} \mathrm{H}_{16}$ & 1005 & B. multifida & [45] \\
\hline
\end{tabular}


Table 2. Cont.

\begin{tabular}{|c|c|c|c|c|c|}
\hline No. & Compound Name & Molecular Formula & Retention Indices (RI) & Sources & References \\
\hline 9 & $\alpha$-terpinene & $\mathrm{C}_{10} \mathrm{H}_{16}$ & 1018 & B. multifida & [45] \\
\hline 10 & p-cymene & $\mathrm{C}_{10} \mathrm{H}_{14}$ & 1025 & B. heterostemon & [48] \\
\hline 11 & limonene & $\mathrm{C}_{10} \mathrm{H}_{16}$ & 1029 & $\begin{array}{l}\text { B. multifida } \\
\text { B. heterostemon }\end{array}$ & {$[45,48]$} \\
\hline 12 & 1,8-cineole & $\mathrm{C}_{10} \mathrm{H}_{18} \mathrm{O}$ & 1033 & $\begin{array}{l}\text { B. multifida } \\
\text { B. heterostemon }\end{array}$ & {$[45,47,48]$} \\
\hline 13 & trans- $\beta$-ocimene & $\mathrm{C}_{10} \mathrm{H}_{16}$ & 1050 & B. heterostemon & [48] \\
\hline 14 & $\gamma$-terpinene & $\mathrm{C}_{10} \mathrm{H}_{16}$ & 1062 & $\begin{array}{l}\text { B. multifida } \\
\text { B. heterostemon }\end{array}$ & {$[45,48]$} \\
\hline 15 & trans-sabinene hydrate & $\mathrm{C}_{10} \mathrm{H}_{18} \mathrm{O}$ & 1064 & B. multifida & {$[44,45]$} \\
\hline 16 & linalool & $\mathrm{C}_{10} \mathrm{H}_{18} \mathrm{O}$ & 1099 & $\begin{array}{l}\text { B. multifida } \\
\text { B. heterostemon }\end{array}$ & {$[44,45,48]$} \\
\hline 17 & nonanal & $\mathrm{C}_{9} \mathrm{H}_{18} \mathrm{O}$ & 1102 & B. multifida & [45] \\
\hline 18 & octyl acetate & $\mathrm{C}_{10} \mathrm{H}_{20} \mathrm{O}_{2}$ & 1124 & B. multifida & [45] \\
\hline 19 & cis-limonene oxide & $\mathrm{C}_{10} \mathrm{H}_{16} \mathrm{O}$ & 1131 & B. orphanidis & [43] \\
\hline 20 & trans-pinocarveol & $\mathrm{C}_{10} \mathrm{H}_{16} \mathrm{O}$ & 1140 & B. multifida & [45] \\
\hline 21 & camphor & $\mathrm{C}_{10} \mathrm{H}_{16} \mathrm{O}$ & 1143 & $\begin{array}{l}\text { B. multifida } \\
\text { B. heterostemon }\end{array}$ & {$[44,47,48]$} \\
\hline 22 & (Z)-3-nonenol & $\mathrm{C}_{9} \mathrm{H}_{18} \mathrm{O}$ & 1158 & B. multifida & [47] \\
\hline 23 & pinocarvone & $\mathrm{C}_{10} \mathrm{H}_{14} \mathrm{O}$ & 1164 & B. multifida & [45] \\
\hline 24 & borneol & $\mathrm{C}_{10} \mathrm{H}_{18} \mathrm{O}$ & 1168 & B. multifida & [47] \\
\hline 25 & terpinen-4-ol & $\mathrm{C}_{10} \mathrm{H}_{18} \mathrm{O}$ & 1177 & B. multifida & [45] \\
\hline 26 & $\alpha$-terpineol & $\mathrm{C}_{10} \mathrm{H}_{18} \mathrm{O}$ & 1189 & $\begin{array}{l}\text { B. multifida } \\
\text { B. orphanidis }\end{array}$ & {$[43,45,47]$} \\
\hline 27 & myrtenal & $\mathrm{C}_{10} \mathrm{H}_{14} \mathrm{O}$ & 1197 & B. multifida & [45] \\
\hline 28 & decanal & $\mathrm{C}_{10} \mathrm{H}_{20} \mathrm{O}$ & 1204 & B. multifida & [47] \\
\hline 29 & trans-carveol & $\mathrm{C}_{10} \mathrm{H}_{16} \mathrm{O}$ & 1217 & B. multifida & [45] \\
\hline 30 & carvone & $\mathrm{C}_{10} \mathrm{H}_{14} \mathrm{O}$ & 1242 & $\begin{array}{l}\text { B. multifida } \\
\text { B. heterostemon }\end{array}$ & {$[45,47,48]$} \\
\hline 31 & geraniol & $\mathrm{C}_{10} \mathrm{H}_{18} \mathrm{O}$ & 1255 & B. heterostemon & [48] \\
\hline 32 & linalyl acetate & $\mathrm{C}_{12} \mathrm{H}_{20} \mathrm{O}_{2}$ & 1257 & B. orphanidis & [43] \\
\hline 33 & isobornyl acetate & $\mathrm{C}_{12} \mathrm{H}_{20} \mathrm{O}_{2}$ & 1283 & B. multifida & [47] \\
\hline 34 & bornyl acetate & $\mathrm{C}_{12} \mathrm{H}_{20} \mathrm{O}_{2}$ & 1285 & B. multifida & [45] \\
\hline 35 & thymol & $\mathrm{C}_{10} \mathrm{H}_{14} \mathrm{O}$ & 1290 & B. multifida & [45] \\
\hline 36 & $(2 E, 4 Z)$-decadienal & $\mathrm{C}_{10} \mathrm{H}_{16} \mathrm{O}$ & 1293 & B. multifida & [47] \\
\hline 37 & carvacrol & $\mathrm{C}_{10} \mathrm{H}_{14} \mathrm{O}$ & 1302 & B. multifida & [47] \\
\hline 38 & $(2 E, 4 E)$-decadienal & $\mathrm{C}_{10} \mathrm{H}_{16} \mathrm{O}$ & 1316 & B. multifida & [47] \\
\hline 39 & $\delta$-elemene & $\mathrm{C}_{15} \mathrm{H}_{24}$ & 1339 & B. heterostemon & [48] \\
\hline 40 & $\alpha$-longipinene & $\mathrm{C}_{15} \mathrm{H}_{24}$ & 1352 & B. heterostemon & [48] \\
\hline 41 & eugenol & $\mathrm{C}_{10} \mathrm{H}_{12} \mathrm{O}_{2}$ & 1361 & B. multifida & [47] \\
\hline 42 & $\alpha$-ylangene & $\mathrm{C}_{15} \mathrm{H}_{24}$ & 1374 & B. multifida & [47] \\
\hline 43 & geranyl acetate & $\mathrm{C}_{12} \mathrm{H}_{20} \mathrm{O}_{2}$ & 1381 & $\begin{array}{l}\text { B. heterostemon } \\
\text { B. multifida }\end{array}$ & [48] \\
\hline 44 & $\beta$-elemene & $\mathrm{C}_{15} \mathrm{H}_{24}$ & 1391 & $\begin{array}{l}\text { B. orphanidis } \\
\text { B. heterostemon }\end{array}$ & {$[43,45,48]$} \\
\hline 45 & tetradecane & $\mathrm{C}_{14} \mathrm{H}_{30}$ & 1400 & B. multifida & [46] \\
\hline 46 & isocaryophyllene & $\mathrm{C}_{15} \mathrm{H}_{24}$ & 1408 & B. multifida & [47] \\
\hline 47 & cis-caryophyllene & $\mathrm{C}_{15} \mathrm{H}_{24}$ & 1409 & B. heterostemon & [48] \\
\hline 48 & $\alpha$-gurjunene & $\mathrm{C}_{15} \mathrm{H}_{24}$ & 1412 & $\begin{array}{l}\text { B. orphanidis } \\
\text { B. multifida }\end{array}$ & [43] \\
\hline 49 & $\beta$-caryophyllene & $\mathrm{C}_{15} \mathrm{H}_{24}$ & 1416 & $\begin{array}{l}\text { B. orphanidis } \\
\text { B. heterostemon }\end{array}$ & {$[43-45,47,48]$} \\
\hline 50 & $\alpha$-bergamotene & $\mathrm{C}_{15} \mathrm{H}_{24}$ & 1418 & B. heterostemon & [48] \\
\hline 51 & $\beta$-duprezianene & $\mathrm{C}_{15} \mathrm{H}_{24}$ & 1424 & B. multifida & [47] \\
\hline 52 & $\gamma$-elemene & $\mathrm{C}_{15} \mathrm{H}_{24}$ & 1431 & $\begin{array}{l}\text { B. multifida } \\
\text { B. heterostemon }\end{array}$ & {$[45,48]$} \\
\hline 53 & $\alpha$-humulene & $\mathrm{C}_{15} \mathrm{H}_{24}$ & 1449 & $\begin{array}{l}\text { B. multifida } \\
\text { B. heterostemon }\end{array}$ & {$[45,48]$} \\
\hline 54 & $\beta$-farnesene & $\mathrm{C}_{15} \mathrm{H}_{24}$ & 1457 & $\begin{array}{l}\text { B. multifida } \\
\text { B. heterostemon }\end{array}$ & {$[44,45,47,48]$} \\
\hline
\end{tabular}


Table 2. Cont.

\begin{tabular}{|c|c|c|c|c|c|}
\hline No. & Compound Name & Molecular Formula & Retention Indices (RI) & Sources & References \\
\hline 55 & allo-aromadendrene & $\mathrm{C}_{15} \mathrm{H}_{24}$ & 1462 & $\begin{array}{l}\text { B. multifida } \\
\text { B. heterostemon }\end{array}$ & {$[44,48]$} \\
\hline 56 & $\alpha$-amorphene & $\mathrm{C}_{15} \mathrm{H}_{24}$ & 1480 & B. heterostemon & [48] \\
\hline 57 & germacrene D & $\mathrm{C}_{15} \mathrm{H}_{24}$ & 1485 & $\begin{array}{l}\text { B. multifida } \\
\text { B. heterostemon }\end{array}$ & {$[45,48]$} \\
\hline 58 & $(E)$ - $\beta$-ionone & $\mathrm{C}_{13} \mathrm{H}_{20} \mathrm{O}$ & 1486 & B. multifida & [47] \\
\hline 59 & cis- $\beta$-guaiene & $\mathrm{C}_{15} \mathrm{H}_{24}$ & 1487 & B. heterostemon & [48] \\
\hline 60 & epi-cubebol & $\mathrm{C}_{15} \mathrm{H}_{26} \mathrm{O}$ & 1495 & B. multifida & [47] \\
\hline 61 & bicyclogermacrene & $\mathrm{C}_{15} \mathrm{H}_{24}$ & 1495 & B. multifida & [45] \\
\hline 62 & $\alpha$-selinene & $\mathrm{C}_{15} \mathrm{H}_{24}$ & 1498 & B. heterostemon & [48] \\
\hline 63 & germacrene A & $\mathrm{C}_{15} \mathrm{H}_{24}$ & 1501 & B. heterostemon & [48] \\
\hline 64 & $\beta$-bisabolene & $\mathrm{C}_{15} \mathrm{H}_{24}$ & 1505 & B. multifida & [47] \\
\hline 65 & $\alpha$-farnesene & $\mathrm{C}_{15} \mathrm{H}_{24}$ & 1507 & B. multifida & [44] \\
\hline 66 & $\gamma$-cadinene & $\mathrm{C}_{15} \mathrm{H}_{24}$ & 1512 & B. multifida & {$[44,47]$} \\
\hline 67 & $\delta$-cadinene & $\mathrm{C}_{15} \mathrm{H}_{24}$ & 1522 & B. multifida & {$[44,45,47]$} \\
\hline 68 & d-cadinene & $\mathrm{C}_{15} \mathrm{H}_{24}$ & 1525 & B. heterostemon & {$[48]$} \\
\hline 69 & guaia-3,9-diene & $\mathrm{C}_{15} \mathrm{H}_{24}$ & 1534 & B. heterostemon & [48] \\
\hline 70 & $\alpha$-cadinene & $\mathrm{C}_{15} \mathrm{H}_{24}$ & 1536 & $\begin{array}{l}\text { B. multifida } \\
\text { B. heterostemon }\end{array}$ & {$[44,48]$} \\
\hline 71 & nerolidol & $\mathrm{C}_{15} \mathrm{H}_{26} \mathrm{O}$ & 1538 & $\begin{array}{l}\text { B. multifida } \\
\text { B. heterostemon }\end{array}$ & {$[45,48]$} \\
\hline 72 & eudesma-3,7(11)-diene & $\mathrm{C}_{15} \mathrm{H}_{24}$ & 1545 & B. heterostemon & [48] \\
\hline 73 & elemol & $\mathrm{C}_{15} \mathrm{H}_{26} \mathrm{O}$ & 1552 & B. multifida & [44] \\
\hline 74 & elixene & $\mathrm{C}_{15} \mathrm{H}_{24}$ & 1559 & B. heterostemon & [48] \\
\hline 75 & germacrane B & $\mathrm{C}_{15} \mathrm{H}_{24}$ & 1563 & B. orphanidis & [43] \\
\hline 76 & $(E)$-nerolidol & $\mathrm{C}_{15} \mathrm{H}_{26} \mathrm{O}$ & 1565 & B. multifida & [44-47] \\
\hline 77 & spathulenol & $\mathrm{C}_{15} \mathrm{H}_{24} \mathrm{O}$ & 1578 & $\begin{array}{l}\text { B. multifida } \\
\text { B. orphanidis } \\
\text { B. multifida }\end{array}$ & {$[43,45,47]$} \\
\hline 78 & caryophyllene oxide & $\mathrm{C}_{15} \mathrm{H}_{24} \mathrm{O}$ & 1583 & $\begin{array}{l}\text { B. orphanidis } \\
\text { B. heterostemon }\end{array}$ & {$[43-45,47,48]$} \\
\hline 79 & viridiflorol & $\mathrm{C}_{15} \mathrm{H}_{26} \mathrm{O}$ & 1594 & B. multifida & [44] \\
\hline 80 & hexadecane & C16H34 & 1600 & B. multifida & [46] \\
\hline 81 & guaiol & $\mathrm{C}_{15} \mathrm{H}_{26} \mathrm{O}$ & 1601 & B. multifida & [47] \\
\hline 82 & humulene epoxide II & $\mathrm{C}_{15} \mathrm{H}_{24} \mathrm{O}$ & 1610 & B. multifida & [44] \\
\hline 83 & $\beta$-elemenone & $\mathrm{C}_{15} \mathrm{H}_{22} \mathrm{O}$ & 1612 & B. heterostemon & [48] \\
\hline 84 & dillapiole & $\mathrm{C}_{12} \mathrm{H}_{14} \mathrm{O}_{4}$ & 1624 & B. multifida & [47] \\
\hline 85 & $\tau$-cadinol & $\mathrm{C}_{15} \mathrm{H}_{26} \mathrm{O}$ & 1635 & B. multifida & {$[44,45]$} \\
\hline 86 & epi- $\alpha$-cadinol & $\mathrm{C}_{15} \mathrm{H}_{26} \mathrm{O}$ & 1642 & $\begin{array}{l}\text { B. multifida } \\
\text { B. orphanidis }\end{array}$ & {$[43,47]$} \\
\hline 87 & $\alpha$-eudesmol & $\mathrm{C}_{15} \mathrm{H}_{26} \mathrm{O}$ & 1656 & B. multifida & {$[44,47]$} \\
\hline 88 & $\alpha$-bisabolol oxide B & $\mathrm{C}_{15} \mathrm{H}_{26} \mathrm{O}_{2}$ & 1658 & B. orphanidis & {$[43]$} \\
\hline 89 & bulnesol & $\mathrm{C}_{15} \mathrm{H}_{26} \mathrm{O}$ & 1671 & B. multifida & {$[44,47]$} \\
\hline 90 & (Z)- $\alpha$-santalol & $\mathrm{C}_{15} \mathrm{H}_{24} \mathrm{O}$ & 1674 & B. orphanidis & {$[43]$} \\
\hline 91 & cis- $\beta$-elemenone & $\mathrm{C}_{15} \mathrm{H}_{22} \mathrm{O}$ & 1678 & $\begin{array}{l}\text { B. heterostemon } \\
\text { B. multifida }\end{array}$ & [48] \\
\hline 92 & $\alpha$-bisabolol & $\mathrm{C}_{15} \mathrm{H}_{26} \mathrm{O}$ & 1688 & $\begin{array}{l}\text { B. orphanidis } \\
\text { B. heterostemon }\end{array}$ & {$[43,44,47,48]$} \\
\hline 92 & germacrone & $\mathrm{C}_{15} \mathrm{H}_{22} \mathrm{O}$ & 1699 & B. heterostemon & [48] \\
\hline 94 & (E)-nerolidol acetate & $\mathrm{C}_{15} \mathrm{H}_{26} \mathrm{O}$ & 1714 & B. multifida & {$[44,47]$} \\
\hline 95 & $(2 E, 6 E)$-farnesol & $\mathrm{C}_{15} \mathrm{H}_{26} \mathrm{O}$ & 1727 & B. multifida & {$[44,47]$} \\
\hline 96 & octadecane & $\mathrm{C}_{18} \mathrm{H}_{38}$ & 1800 & B. multifida & {$[46,47]$} \\
\hline 97 & neophytadiene & $\mathrm{C}_{20} \mathrm{H}_{38}$ & 1836 & B. multifida & {$[46]$} \\
\hline 98 & 6,10,14-trimethyl-2-pentadecanone & $\mathrm{C}_{18} \mathrm{H}_{36} \mathrm{O}$ & 1845 & B. multifida & {$[44,46,47]$} \\
\hline 99 & nonadecane & $\mathrm{C}_{19} \mathrm{H}_{40}$ & 1900 & B. multifida & [47] \\
\hline 100 & farnesyl acetone & $\mathrm{C}_{18} \mathrm{H}_{30} \mathrm{O}$ & 1917 & B. multifida & {$[44,47]$} \\
\hline 101 & methyl linolenate & $\mathrm{C}_{19} \mathrm{H}_{32} \mathrm{O}_{2}$ & 2098 & B. multifida & [47] \\
\hline 102 & phytol & $\mathrm{C}_{20} \mathrm{H}_{40} \mathrm{O}$ & 2124 & B. multifida & {$[44,47]$} \\
\hline 104 & mandenol & $\mathrm{C}_{20} \mathrm{H}_{36} \mathrm{O}_{2}$ & 2148 & B. multifida & {$[44]$} \\
\hline 104 & ethyl linolenate & $\mathrm{C}_{20} \mathrm{H}_{34} \mathrm{O}_{2}$ & 2162 & B. multifida & {$[44,47]$} \\
\hline 105 & 10-cyclohexyl-nonadecane & $\mathrm{C}_{25} \mathrm{H}_{52}$ & 2312 & B. multifida & {$[44]$} \\
\hline
\end{tabular}


Table 2. Cont.

\begin{tabular}{lccccc}
\hline No. & Compound Name & Molecular Formula & Retention Indices (RI) & Sources & References \\
\hline 106 & pentacosane & $\mathrm{C}_{25} \mathrm{H}_{52}$ & 2517 & B. multifida & {$[44]$} \\
107 & $n$-heptacosane & $\mathrm{C}_{27} \mathrm{H}_{56}$ & 2682 & B. multifida & {$[44]$} \\
108 & octacosane & $\mathrm{C}_{28} \mathrm{H}_{58}$ & 2791 & B. multifida & {$[44]$} \\
109 & nonacosane & $\mathrm{C}_{29} \mathrm{H}_{60}$ & 2894 & B. multifida & {$[44]$} \\
110 & vitamin E & $\mathrm{C}_{29} \mathrm{H}_{50} \mathrm{O}_{2}$ & 3138 & B. multifida & {$[44]$} \\
111 & $n$-docosane & $\mathrm{C}_{22} \mathrm{H}_{46}$ & - & B. multifida & {$[44]$} \\
112 & epizonaren & $\mathrm{C}_{15} \mathrm{H}_{24}$ & - & B. multifida & [44] \\
\hline
\end{tabular}

\section{Applications in Traditional Medicines}

Among the four well-known Biebersteinia spp., only B. heterostemon and B. multifida have been commonly applied as traditional herbal medicines to treat musculoskeletal disorders, bone fractures and skin diseases $[7,25,55]$. In China, B. heterostemon plants are widely distributed in Qinghai-Tibetan Plateau, and have been administered as traditional Tibetan medicines [20,56]. In addition, B. multifida is indigenous to Iran, where this plant species has been topically applied as a folk remedy for treatments of muscle and skeletal disorders and bone fractures [9,57]. Besides, it has also been reported that children's nocturia can be treated with B. multifida [58]. In addition, B. odora has been used in treatments of migraine and fever for centuries by people living in the Shigar Valley, Baltistan region of Karakorum range, Pakistan [8]. As Biebersteinia species have high pharmacological values as traditional medicines, their bioactivities have attracted the attention of a large number of phytochemists and pharmacologists.

\section{Pharmacological Activities}

\subsection{In Vivo Pharmacological Activities}

\subsubsection{Anti-Inflammatory and Analgesic Effects}

The anti-inflammatory effect of $B$. heterostemon has been evaluated with a xylene-induced murine inflammation model. Its analgesic effect on mice was established by the hotplate and tail flick methods and by acetic acid-induced writhing [55]. Traditional B. heterostemon decoctions, traditional $B$. heterostemon decoctions followed by alcohol precipitation, and ethanolic B. heterostemon extracts inhibited xylene-induced ear edema in mice and elevated the mouse hotplate pain threshold [55]. However, the anti-inflammatory and analgesic efficacies of the ethanolic B. heterostemon extract were significantly stronger than those of the other afore-mentioned extracts [55]. These results might be correlated with those for N-3-methyl-2-butenyl urea (40) isolated from the ethanolic extract of $B$. heterostemon, as this compound was confirmed to have analgesic activity [11]. Similar findings were obtained and reported for B. multifida. A dose of $10 \mathrm{mg} / \mathrm{kg}$ B. multifida root extract obtained by ethanol refluxing, and that of $4 \mathrm{mg} / \mathrm{kg}$ indomethacin had similar anti-inflammatory efficacies in a carrageenan-induced edema assay [57]. The first phase of a formalin test indicated that the analgesic efficacy of $50 \mathrm{mg} / \mathrm{kg}$ B. multifida root extract was comparable to that of $10 \mathrm{mg} / \mathrm{kg}$ morphine [57]. These findings collectively indicate the high potential of B. heterostemon and B. multifida for the production of anti-inflammatory and analgesic drugs.

\subsubsection{Anti-Hypertensive and Hypoglycemic Effects}

The compound N-3-methyl-2-butenyl urea (40) isolated from B. heterostemon displayed both analgesic and antihypertensive activities [11]. Numerous alkaloids from natural resources exhibited hypoglycemic effects [14]. B. heterostemon alkaloids showed significant hypoglycemic efficacy in streptozotocin-induced diabetic mice, with the optimum therapeutic dose at $5 \mathrm{mg} / \mathrm{kg}$ [59]. On the other hand, neither antihypertensive nor hypoglycemic activity was detected in any other Biebersteinia species. In addition, galegine (30), an isopentenyl guanidine, which was originally isolated from 
Galega officinalis and has significant hypoglycemic activity [60], was also detected in B. heterostemon [25]. In fact, the hypoglycemic drug metformin is a derivative of galegine [61-64].

\subsubsection{Anti-Fatigue and Anxiolytic Effects}

The anti-fatigue effect of $B$. multifida root extract was also validated in a forced swimming test (FST), and the biochemical parameters in the blood related to fatigue were measured [7]. The results demonstrated the potential benefit of B. multifida root extract as an anti-fatigue material, and showed that it improved physical stamina [7]. These properties and effects might account for the fact that B. multifida has been used in Iranian folk medicine to enhance physical strength [10]. In addition, B. multifida total root extracts exhibited anxiolytic effect in an elevated plus-maze assay [23]. This finding led to the isolation and characterization of three active coumarin derivatives from B. multifida root extracts, namely umbelliferone (33), scopoletin (34) and ferulic acid (35) with the well-known potent monoamine oxidase (MAO) inhibitory and anti-anxiety effects $[23,65,66]$. These discoveries explain and provide scientific evidence to support the traditional use of B. multifida for the management of anxiety.

\subsubsection{Hypolipidemic Effect}

It has been well-established that lipoproteins play vital roles in atherosclerosis $[67,68]$. Over the past several decades, a number of studies have indicated that low-density lipoproteins (LDL) and high-density lipoproteins (HDL) have opposite influences as risk factors in the onset and progression of atherosclerosis [67,69-73]. It was verified in the last 20 years that lowering LDL-cholesterol successfully prevents atherosclerosis [74]. B. multifida root extracts, prepared by using a solution of water and ethanol with the ratio of 1:2, significantly reduced both the HDL and LDL levels in mice serum at doses of 4 and $5 \mathrm{mg} / \mathrm{kg}$, respectively [75]. In addition, the hydro-methanolic extract of B. multifida roots was recently observed to possess a protective effect on ethanol-induced gastric ulcer in rats, which was thought to be partly related to antioxidant activity and accelerating nitric oxide (NO) production in vivo after the rats were treated with the extracts [76]. Taken together, Biebersteinia species can be explored for a wide range of pharmacological activities.

\subsection{In Vitro Pharmacological Activities}

\subsubsection{Antimicrobial Effects}

A number of studies have reported that B. heterostemon and B. multifida extracts significantly inhibited the growth of various bacteria and fungi in a concentration-dependent manner. For instance, the whole plant extracts of $B$. heterostemon substantially inhibited the growth and proliferation of the pathogenic fungi Fusarium equiseti, F. oxysporum and F. moniliforme, which are thought to be the causes of inducing the Chinese Angelica stem nematode disease, with the minimum inhibitory concentrations (MICs) of $0.6250 \mathrm{mg} / \mathrm{mL}, 0.6250 \mathrm{mg} / \mathrm{mL}$ and $1.2500 \mathrm{mg} / \mathrm{mL}$, respectively [77]. Another independent study estimated the antibacterial activities of root extracts from B. multifida against various Gram-positive or negative bacteria, including Bacillus cereus, Clostridium perfringens, Staphylococcus aureus, Escherichia coli, Enterobacter aerogenes and Salmonella enterica [78]. The results unraveled that the root extracts of B. multifida obtained by $n$-hexane and ethanol maceration displayed significant antibacterial effects with the MIC of $0.195 \mathrm{mg} / \mathrm{mL}$ [78]. Some terpene compounds isolated from B. heterostemon were confirmed to possess antibacterial activities. Moreover, the compound (-)-anymol-8-O- $\beta$-D-lyxopyranoside (38), a bisabolane-type sesquiterpene glycoside isolated from $B$. heterostemon, displayed a pronounced antibacterial efficacy against B. subtilis, S. aureus and Pseudomonas spp. with the MICs of $50 \mu \mathrm{g} / \mathrm{mL}$, $50 \mu \mathrm{g} / \mathrm{mL}$ and $70 \mu \mathrm{g} / \mathrm{mL}$, respectively [26]. In addition, the prenylated guanidine known as galegine (30) was reported to exhibit the most potent antibacterial efficacy against various S. aureus strains, including the two methicillin-resistant ones, in the concentration range between 20 and $31 \mu \mathrm{M}[79,80]$. 


\subsubsection{Antioxidant Activities}

Numerous studies have reported on the antioxidant activities of dietary phenolic substances like flavonoids in various living organisms, including plants, animals and humans [81-86]. Most of the published reports have focused on the antioxidant activities of phenolics possessing the ability to inhibit the formation of free radicals, the mode of which depends mainly on the structure-activity relationships of antioxidant compounds [87-90]. High CTF in Biebersteinia plants is closely correlated with their antioxidant activities [17]. Various methods, including 1,1-diphenyl-2-picrylhydrazyl (DPPH) and 2,2'-azinobis(3-ethylbenzothiazoline-6-sulfonic acid) (ABTS) radical-scavenging approaches, and Oil Stability Index (OSI) assay, have been used to determine the antioxidant activities of different B. heterostemon solvent extracts, and disclosed that their antioxidant activities varied considerably [91]. Specifically, the ethyl acetate and ethanolic extracts of $B$. heterostemon aerial parts were presented with higher antioxidant activities than the $n$-hexane extract, and their relative efficacies were concentration-dependent [91]. One possible explanation is that flavonoids and phenols were more readily extracted with polar than nonpolar solvents [92-94]. In addition, a B. multifida root extract was found to be enriched with phenolic compounds $(80.1 \pm 3.10 \mathrm{mg} / \mathrm{mL})$, and demonstrated strong DPPH radical-scavenging activity $(95.9 \pm 3.20 \mu \mathrm{g} / \mathrm{mL})$ [21]. It is worth mentioning that the polyphenolic compounds identified in food products prepared from various plant sources like Avena sativa, Aristotelia chilensis, Paeonia ostii and Linum usitatissimum possess significant antioxidant activities as well [95-98].

Besides antioxidant activities related to phenolic compounds present in Biebersteinia spp., the essential oils from different types of tissues of Biebersteinia plants were also shown to have strong radical-scavenging activities. For instance, the essential oil of B. multifida fruits, evaluated by DPPH assay, was shown to be superior to essential oils extracted from other organs (e.g., leaves and roots), displaying the $\mathrm{IC}_{50}$ value of $16.7 \pm 0.02 \mu \mathrm{g} / \mathrm{mL}$ that was even more excellent than the well-known synthetic antioxidant butylated hydroxytoluene (BHT, $19.0 \pm 0.80 \mu \mathrm{g} / \mathrm{mL}$ ) [45]. The chemical composition in the essential oils of $B$. multifida fruits should be responsible for their antioxidant activity due to the antioxidative properties of thymol, 1,8-cineol and $\beta$-caryophyllene [45], which are the major compounds in B. multifida essential oils $[99,100]$. These investigations indicate that the Biebersteinia species are a valuable natural resource for extracting antioxidant compounds.

\subsubsection{Anti-Cancer Effects}

A growing body of literature has demonstrated that Biebersteinia species possess some other valuable pharmacological effects, in addition to those overviewed in previous subsections. For instance, an ethanolic extract from B. multifida roots was reported to prevent mutation reversion by $51.2 \%$ in an anti-mutagenicity test, indicating that $B$. multifida plants harbor natural products that can act as anticancer agents [101]. Another independent study showed that the root extract of $B$. multifida obtained by maceration with $70 \%$ ethanol was cytotoxic and apoptotic to both human prostate cancer cells DU145 and PC3 in a dose-dependent manner, as this extract significantly decreased cell viability [102].

\section{Conclusions and Future Perspectives}

Natural plant products have been used extensively and widely in traditional medicine, and are important sources for drug discovery and development. Up-to-date, only a few studies have examined and analyzed the phytochemical constituents, bioactivities, and pharmacological aspects and characteristics of Biebersteinia species. More than 40 secondary metabolites have been isolated and identified in the members of this plant genus, of which flavonoids were the principal constituents. The varied properties and efficacies of the pharmacologically active substances in different Biebersteinia species suggest that these compounds are potential sources of new drugs.

However, certain key issues must be resolved before the identified Biebersteinia species can be fully exploited as bases for new pharmaceutical agents. Currently, many of their phytochemical constituents have not yet been systematically identified, and some of those that have already been elucidated do 
not necessarily account for their observed pharmacological effects. Although certain constituents have significant pharmaceutical effects, their underlying mode-of-action and molecular mechanisms remain unclear. Moreover, in vivo and in vitro models should be designed and implemented in order to screen for unrecognized bioactivities. For instance, although B. heterostemon is widely used in folk medicine in northwest China, it has also generally been regarded as toxic, or a weed that is difficult to be eradicated. Consequently, the potential utility of this resource has been underexploited, or even was lost altogether. In order to harness the full value of the identified Biebersteinia species as pharmaceutical agents, we should perform basic research on their bioactive constituents, pharmacological properties and molecular mechanisms, which is followed by clinical tests. In-depth investigations are therefore required to develop, test and optimize the administration of novel drugs derived from various organs of plants of this genus. Overall, we believe that this synopsis will facilitate the development and exploitation of new drug resources from these plant materials.

Author Contributions: All authors listed have made substantial direct and intellectual contributions to the work, and approved it for publication. All authors have read and agreed to the published version of this manuscript.

Funding: This work was financially supported by the Natural Science Foundation of Qinghai Province (Nos. 2017-ZJ-943Q and 2017-ZJ-940Q), the National Natural Science Foundation of China (Nos. 81760633 and 31500049), the Open Project of the State Key Laboratory of Plateau Ecology and Agriculture of Qinghai University (No. 2017-KF-04), and the Qinghai Innovation Platform Construction Project (No. 2017-ZJ-Y20).

Conflicts of Interest: The authors declare no conflicts of interest.

\section{References}

1. Liu, J.Q.; Ho, T.N.; Chen, S.L.; Lu, A. Karyomorphology of Biebersteinia Stephan (Geraniaceae) and its systematic and taxonomic significance. Bot. Bull. Acad. Sin. 2001, 42, 61-66.

2. Bakker, F.T.; Vassiliades, D.D.; Morton, C. Phylogenetic relationships of Biebersteinia Stephan (Geraniaceae) inferred from $r b c L$ and $a t p B$ sequence comparisons. Bot. J. Linn. Soc. 1998, 127, 149-158. [CrossRef]

3. Muellner, A.N.; Vassiliades, D.D.; Renner, S.S. Placing Biebersteiniaceae, a herbaceous clade of Sapindales, in a temporal and geographic context. Plant Syst. Evol. 2007, 266, 233-252. [CrossRef]

4. Christenhusz, M.J.M.; Byng, J.W. The number of known plants species in the world and its annual increase. Phytotaxa 2016, 261, 201-217. [CrossRef]

5. Tan, K.; Perdetzoglou, D.K.; Roussis, V. Biebersteinia orphanidis (Geraniaceae) from southern Greece. Ann. Bot. Fenn. 1997, 34, 41-45.

6. Yannitsaros, A.G.; Constantinidis, T.A.; Vassiliades, D.D. The rediscovery of Biebersteinia orphanidis Boiss. (Geraniaceae) in Greece. Bot. J. Linn. Soc. 1996, 120, 239-242. [CrossRef]

7. Jahromy, M.; Mohajer, A.; Adibi, L.; Khakpour, S. Effects of Biebersteinia multifida DC. Root extract on physical stamina in male mice. Food Nutr. Sci. 2015, 6, 326-331. [CrossRef]

8. Doležal, J.; Dvorský, M.; Börner, A.; Wild, J.; Schweingruber, F.H. Anatomical descriptions of dicotyledons. In Anatomy, Age and Ecology of High Mountain Plants in Ladakh, the Western Himalaya; Springer International Publishing: Cham, Switzerland, 2018.

9. Abbas, Z.; Khan, S.M.; Alam, J.; Khan, S.W.; Abbasi, A.M. Medicinal plants used by inhabitants of the Shigar Valley, Baltistan region of Karakorum range-Pakistan. J. Ethnobiol. Ethnomed. 2017, 13, 53. [CrossRef]

10. Amirghofran, Z. Medicinal plants as immunosuppressive agents in traditional Iranian medicine. Iran J. Immunol. 2010, 7, 65-73.

11. Zhang, X.F.; Hu, B.L.; Zhou, B.N. Studies on the active constituents of Tibetan herb Biebersteinia heterostemon Maxim. Acta Pharm. Sin. 1995, 30, 211-214.

12. Wang, W.E.; Zhang, X.F.; Shen, J.W.; Lou, D.J. Chemical constituents in aerial parts of Biebersteinia heterostemon Maxim. Nat. Prod. Res. Develop. 2009, 21, 199-202.

13. Zhang, P.Z.; Zhong, G.Y.; Xie, W.W.; Zhang, Y.M. Flavonoids from Biebersteinia heterostemon. Chin. Tradit. Herb. Drugs 2016, 47, 3565-3568. (In Chinese with English abstract)

14. Zhang, Y.H.; Zhang, D.J.; Zhang, B.Y. Chemical structures of alkaloids with hypoglycemic activity and their hypoglycemic mechanisms. Chin. Tradit. Herb. Drugs 2018, 49, 3692-3702. (In Chinese with English abstract) 
15. Greenham, J.; Vassiliades, D.D.; Harborne, J.B.; Williams, C.A.; Eagles, J.; Grayer, R.J.; Veitch, N.C. A distinctive flavonoid chemistry for the anomalous genus Biebersteinia. Phytochemistry 2001, 56, 87-91. [CrossRef]

16. Bate-Smith, E.C. Chemotaxonomy of Geranium. Bot. J. Linn. Soc. 1973, 67, 347-359. [CrossRef]

17. Duda, S.C.; Mărghitaş, L.A.; Dezmirean, D.; Duda, M.; Mărgăoan, R.; Bobiş, O. Changes in major bioactive compounds with antioxidant activity of Agastache foeniculum, Lavandula angustifolia, Melissa officinalis and Nepeta cataria: Effect of harvest time and plant species. Ind. Crops Prod. 2015, 77, 499-507. [CrossRef]

18. Jassbi, A.R.; Zare, S.; Asadollahi, M.; Schuman, M.C. Ecological roles and biological activities of specialized metabolites from the genus Nicotiana. Chem. Rev. 2017, 117, 12227-12280. [CrossRef]

19. Zidorn, C. Secondary metabolites of seagrasses (Alismatales and Potamogetonales; Alismatidae): Chemical diversity, bioactivity, and ecological function. Phytochemistry 2016, 124, 5-28. [CrossRef]

20. Tan, Z. Study on the geographic distribution of Geraniaceae plants in Sichuan. Bull. Bot. Res. 1995, 15, 523-531.

21. Zhang, B.Y.; Wang, H.; Yang, X.Y.; Shen, J.W.; Zhang, X.F. The determination of total flavonoids of Biebersteinia heterostemon from different regions of Qinghai province. Chin. J. Anal. Lab. 2009, 28 (Suppl. 2), 39-41.

22. Nabavi, S.F.; Ebrahimzadeh, M.A.; Nabavi, S.M.; Eslami, B.; Dehpour, A. Antihemolytic and antioxidant activities of Biebersteinia multifida. Eur. Rev. Med. Pharmacol. Sci. 2010, 14, 823-830. [PubMed]

23. Monsef-Esfahani, H.R.; Amini, M.; Goodarzi, N.; Saiedmohammadi, F.; Hajiaghaee, R.; Faramarzi, M.A.; Tofighi, Z.; Ghahremani, M.H. Coumarin compounds of Biebersteinia multifida roots show potential anxiolytic effects in mice. DARU J. Pharm. Sci. 2013, 21, 51. [CrossRef]

24. Zhang, Y.H.; Zhang, X.; Zhang, B.Y.; Zhang, D.J. Chemical constituents from Tibetan herbal medicines Biebersteinia heterostemon. Chin. Tradit. Herb. Drugs 2019, 50, 1551-1554. (In Chinese with English abstract) [CrossRef]

25. Wu, H.F.; Zhang, X.F.; Deng, Y.; Pan, L.; Ding, L.S. Studies on chemical constituents from bark of Biebersteinia heterostemon. China J. Chin. Mater. Med. 2007, 32, 2141-2143. (In Chinese with English abstract)

26. Meng, J.C.; Lu, H.; Li, H.; Yang, L.; Tan, R.X. A new antibacterial sesquiterpene glycoside and other bioactive compounds from Biebersteinia heterostemon. Spectrosc. Lett. 1999, 32, 1005-1012. [CrossRef]

27. Kurbanov, D.; Zharekeev, B.K. Investigation of the alkaloids of Biebersteinia multifida and Peganum harmala from Karakalpakia. Chem. Nat. Compd. 1974, 10, 715. [CrossRef]

28. Tzakou, O.; Yannitsaros, A.; Vassiliades, D. Investigation of the $C(16: 3) / C(18: 3)$ fatty acid balance in leaf tissues of Biebersteinia orphanidis Boiss. (Biebersteiniaceae). Biochem. Syst. Ecol. 2001, 29, 765-767. [CrossRef]

29. Wang, F.C.; Che, Z.; Qiu, D.; Wei, L. Analysis of fatty acids in Tibetan medicine Biebersteinia heterostemon Maxim. J. Qinghai Norm. Univ. (Nat. Sci. Ed.) 2013, 38-40. (In Chinese with English abstract) [CrossRef]

30. Benn, M.H.; Shustov, G.; Shustova, L.; Majak, W.; Bai, Y.; Fairey, N.A. Isolation and characterization of two guanidines from Galega orientalis Lam. cv. Gale (fodder galega). J. Agric. Food Chem. 1996, 44, 2779-2781. [CrossRef]

31. Pufahl, K.; Schreiber, K. Isolation of a new guanidine derivative from goat's rue, Galega officinalis L. Experientia 1961, 17, 302-303. [CrossRef]

32. Yang, T.C.; Chao, H.F.; Shi, L.S.; Chang, T.C.; Lin, H.C.; Chang, W.L. Alkaloids from Coptis chinensis root promote glucose uptake in C2C12 myotubes. Fitoterapia 2014, 93, 239-244. [CrossRef]

33. Umezawa, K.; Hiroki, A.; Kawakami, M.; Naka, H.; Takei, I.; Ogata, T.; Kojima, I.; Koyano, T.; Kowithayakorn, T.; Pang, H.S.; et al. Induction of insulin production in rat pancreatic acinar carcinoma cells by conophylline. Biomed. Pharmacother. 2003, 57, 341-350. [CrossRef]

34. Tiong, S.H.; Looi, C.Y.; Arya, A.; Wong, W.F.; Hazni, H.; Mustafa, M.R.; Awang, K. Vindogentianine, a hypoglycemic alkaloid from Catharanthus roseus (L.) G. Don (Apocynaceae). Fitoterapia 2015, 102, 182-188. [CrossRef] [PubMed]

35. Shan, M.; Yu, S.; Yan, H.; Guo, S.; Xiao, W.; Wang, Z.; Zhang, L.; Ding, A.; Wu, Q.; Li, S.F.Y. A review on the phytochemistry, pharmacology, pharmacokinetics and toxicology of geniposide, a natural product. Molecules 2017, 22, 1689. [CrossRef]

36. Xiao, W.; Li, S.; Wang, S.; Ho, C.T. Chemistry and bioactivity of Gardenia jasminoides. J. Food Drug Anal. 2017, 25, 43-61. [CrossRef] [PubMed] 
37. Arifkhodzhaev, A.O.; Arifkhodzhaev, K.A.; Kondratenko, E.S. Polysaccharides of saponin-bearing plants. II. Isolation and characterization of the polysaccharides of Biebersteinia multifida. Chem. Nat. Compd. 1985, 21, 714-716. [CrossRef]

38. Arifkhodzhaev, A.O.; Rakhimov, D.A. Polysaccharides of saponin-bearing plants. III. Polysaccharides of the epigeal organs of Biebersteinia multifida. Chem. Nat. Compd. 1986, 22, 719-720. [CrossRef]

39. Arifkhodzhaev, A.O.; Rakhimov, D.A. Polysaccharides of saponin-bearing plants. IV. Structure of glucans A, B, and C of Biebersteinia multifida. Chem. Nat. Compd. 1993, 29, 151-153. [CrossRef]

40. Arifkhodzhaev, A.O.; Rakhimov, D.A. Polysaccharides of saponin-bearing plants. V. Structural investigation of glucans A, B, and C and their oligosaccharides from Biebersteinia multifida plants. Chem. Nat. Compd. 1994, 30, 655-660. [CrossRef]

41. Ruiz-Rodriguez, A.; Reglero, G.; Ibañez, E. Recent trends in the advanced analysis of bioactive fatty acids. J. Pharm. Biomed. Anal. 2010, 51, 305-326. [CrossRef]

42. Ahmadzadeh Sani, T.; Golmakani, E.; Mohammadi, A.; Feyzi, P.; Kamali, H. Optimization of pressurized hot water extraction on the extract yield and antioxidant activity from Biebersteinia multifida DC using a modified supercritical fluid extractor. J. Supercrit. Fluid. 2014, 94, 130-137. [CrossRef]

43. Fakir, H.; Yasar, S.; Erbas, S.; Ozderin, S. Essential oil composition of Biebersteinia orphanidis Boiss. growing in mediterranean region of Turkey. Asian J. Chem. 2011, 23, 3767-3768.

44. Feyzi, P.; Sani, T.A.; Alesheikh, P.; Kamali, H.; Mohammadi, A. Comparative study of essential oils extracted from Biebersteinia multifida DC using hydro-distillation, microwave and solvent extraction. West Indian Med. J. 2016. [CrossRef]

45. Hamzeh, A. Antioxidant activities of the essential oils and extracts of Biebersteinia multifida DC. Herba Pol. 2009, 55, 59-68.

46. Akhlaghi, H.; Shafaghat, A.; Mohammadhosseini, M. Chemical composition of the essential oil from leaves of Biebersteinia multifida DC. growing wild in Iran. J. Essent. Oil Bear. Plants 2009, 12, 365-368. [CrossRef]

47. Javidnia, K.; Miri, R.; Soltani, M.; Khosravi, A.R. Essential oil composition of Biebersteinia multifida DC. (Biebersteiniaceae) from Iran. J. Essent. Oil Res. 2010, 22, 611-612. [CrossRef]

48. Yang, Y. The Study on Essential Oil of Eleven Tibetan Medicines. Master's Thesis, Qinghai Univerisity for Nationalities, Xining, China, 2012.

49. Capuzzo, A.; Maffei, M.E.; Occhipinti, A. Supercritical fluid extraction of plant flavors and fragrances. Molecules 2013, 18, 7194-7238. [CrossRef]

50. Donato, P.; Inferrera, V.; Sciarrone, D.; Mondello, L. Supercritical fluid chromatography for lipid analysis in foodstuffs. J. Sep. Sci. 2017, 40, 361-382. [CrossRef]

51. Fornari, T.; Vicente, G.; Vazquez, E.; Garcia-Risco, M.R.; Reglero, G. Isolation of essential oil from different plants and herbs by supercritical fluid extraction. J. Chromatogr. A 2012, 1250, 34-48. [CrossRef]

52. Hartmann, A.; Ganzera, M. Supercritical fluid chromatography-Theoretical background and applications on natural products. Planta Med. 2015, 81, 1570-1581. [CrossRef]

53. Pourmortazavi, S.M.; Hajimirsadeghi, S.S. Supercritical fluid extraction in plant essential and volatile oil analysis. J. Chromatogr. A 2007, 1163, 2-24. [CrossRef] [PubMed]

54. Wrona, O.; Rafinska, K.; Mozenski, C.; Buszewski, B. Supercritical fluid extraction of bioactive compounds from plant materials. J. AOAC Int. 2017, 100, 1624-1635. [CrossRef] [PubMed]

55. Jing, M.; Li, Y.; Wang, J.J.; Lin, X.Y.; Liu, X.P.; Ren, Y. Pharmacological effect method of different extraction process evaluation about Tibetan medicine Biebersteinia heterostemon Maxim. J. Med. Pharma. Chin. Minorit. 2012, 18, 62-63.

56. Tang, Y.C. On the Affinities and the Role of the Chinese Flora. Acta Bot. Yunnan. 2000, 22, 1-26.

57. Farsam, H.; Amanlou, M.; Reza Dehpour, A.; Jahaniani, F. Anti-inflammatory and analgesic activity of Biebersteinia multifida DC. root extract. J. Ethnopharmacol. 2000, 71, 443-447. [CrossRef]

58. Aboutorabi, H. Ethnobotanic and Phytochemical Study of Plants in Rouin Region. Ph.D. Thesis, Tehran University of Medical Sciences, Tehran, Iran, 2001.

59. Wang, W.E.; Zhao, W.Y. The hypoglycemic effect of total alkanoids of Biebersteinia heterostemon on streptozotocin-induced diabetic mice. Chin. Tradition. Patent Med. 2011, 33, 1584-1586.

60. Rios, J.L.; Francini, F.; Schinella, G.R. Natural products for the treatment of type 2 diabetes mellitus. Planta Med. 2015, 81, 975-994. [CrossRef]

61. Bailey, C.J. Metformin: Historical overview. Diabetologia 2017, 60, 1566-1576. [CrossRef] 
62. Graham, G.G.; Punt, J.; Arora, M.; Day, R.O.; Doogue, M.P.; Duong, J.K.; Furlong, T.J.; Greenfield, J.R.; Greenup, L.C.; Kirkpatrick, C.M.; et al. Clinical pharmacokinetics of metformin. Clin. Pharmacokinet. 2011, 50, 81-98. [CrossRef]

63. Lee, J.S.; Kim, W.S.; Kim, J.J.; Chin, Y.W.; Jeong, H.C.; Choi, J.S.; Min, H.G.; Cha, H.J. Identification of anti-melanogenic natural compounds from Galega officinalis and further drug repositioning. J. Dermatol. Sci 2012, 67, 61-63. [CrossRef]

64. Patade, G.; Marita, A. Metformin: A Journey from countryside to the bedside. J. Obes. Metab. Res. 2014, 1, 127-130. [CrossRef]

65. Jeong, S.H.; Han, X.H.; Hong, S.S.; Hwang, J.S.; Hwang, J.H.; Lee, D.; Lee, M.K.; Ro, J.S.; Hwang, B.Y. Monoamine oxidase inhibitory coumarins from the aerial parts of Dictamnus albus. Arch. Pharmacal Res. 2006, 29, 1119-1124. [CrossRef]

66. Yun, B.S.; Lee, I.K.; Ryoo, I.J.; Yoo, I.D. Coumarins with monoamine oxidase inhibitory activity and antioxidative coumarino-lignans from Hibiscus syriacus. J. Nat. Prod. 2001, 64, 1238-1240. [CrossRef] [PubMed]

67. Ahotupa, M. Oxidized lipoprotein lipids and atherosclerosis. Free Radic. Res. 2017, 51, 439-447. [CrossRef] [PubMed]

68. Orso, E.; Schmitz, G. Lipoprotein(a) and its role in inflammation, atherosclerosis and malignancies. Clin. Res. Cardiol. Suppl. 2017, 12, 31-37. [CrossRef] [PubMed]

69. Aluganti Narasimhulu, C.; Fernandez-Ruiz, I.; Selvarajan, K.; Jiang, X.; Sengupta, B.; Riad, A.; Parthasarathy, S. Atherosclerosis-Do we know enough already to prevent it? Curr. Opin. Pharmacol. 2016, 27, 92-102. [CrossRef]

70. Bandeali, S.; Farmer, J. High-density lipoprotein and atherosclerosis: The role of antioxidant activity. Curr. Atheroscler. Rep. 2012, 14, 101-107. [CrossRef]

71. Hu, J.; Xi, D.; Zhao, J.; Luo, T.; Liu, J.; Lu, H.; Li, M.; Xiong, H.; Guo, Z. High-density lipoprotein and inflammation and its significance to atherosclerosis. Am. J. Med. Sci. 2016, 352, 408-415. [CrossRef]

72. Orekhov, A.N.; Sobenin, I.A. Modified lipoproteins as biomarkers of atherosclerosis. Front Biosci. (Landmark Ed.) 2018, 23, 1422-1444. [CrossRef]

73. Parhofer, K.G. Increasing HDL-cholesterol and prevention of atherosclerosis: A critical perspective. Atheroscler. Suppl. 2015, 18, 109-111. [CrossRef]

74. Baigent, C.; Blackwell, L.; Emberson, J.; Holland, L.E.; Reith, C.; Bhala, N.; Peto, R.; Barnes, E.H.; Keech, A.; Simes, J.; et al. Efficacy and safety of more intensive lowering of LDL cholesterol: A meta-analysis of data from 170,000 participants in 26 randomised trials. Lancet 2010, 376, 1670-1681. [CrossRef] [PubMed]

75. Khakpour, S.; Akhlaghdoust, M.; Naimi, S.; Mirlohi, S.M.J.; Abedian, M.; Seyed Forootan, N.S.; Foroughi, F. Effect of Biebersteinia multifida DC. root extract on cholesterol in mice. Zahedan J. Res. Med. Sci. 2013, 15, 49-51.

76. Raeesi, M.; Eskandari-Roozbahani, N.; Shomali, T. Gastro-protective effect of Biebersteinia multifida root hydro-methanolic extract in rats with ethanol-induced peptic ulcer. Avicenna J. Phytomed. 2019, 9, 410-418. [PubMed]

77. Lu, Y.Z.; Jing, M.; Liu, J.J.; Lu, N.H.; Chen, H.; Chen, Z.J.; Zhang, Y.X. Inhibition of commonly used traditional Chinese medicine mixed on Chinese Angelica stem nematode disease pathogen. J. Tradit. Chin. Vet. Med. 2017, 36, 38-40.

78. Ghodrati, N.; Asili, J.; Mohammadi, S.A.; Fazli-Bazzaz, B.S. Evaluation of antibacterial activities of different roots extracts of Biebersteinia multifida DC. J. North Khorasan Univ. Med. Sci. 2013, 4, 149-154. [CrossRef]

79. Berlinck, R.G.S.; Bertonha, A.F.; Takaki, M.; Rodriguez, J.P.G. The chemistry and biology of guanidine natural products. Nat. Prod. Rep. 2017, 34, 1264-1301. [CrossRef]

80. Coqueiro, A.; Regasini, L.O.; Stapleton, P.; da Silva Bolzani, V.; Gibbons, S. In vitro antibacterial activity of prenylated guanidine alkaloids from Pterogyne nitens and synthetic analogues. J. Nat. Prod. 2014, 77, 1972-1975. [CrossRef]

81. De Oliveira, N.K.S.; Almeida, M.R.S.; Pontes, F.M.M.; Barcelos, M.P.; de Paula da Silva, C.H.T.; Rosa, J.M.C.; Cruz, R.A.S.; da Silva Hage-Melim, L.I. Antioxidant effect of flavonoids present in Euterpe oleracea martius and neurodegenerative diseases: A literature review. Cent. Nerv. Syst. Agents Med. Chem. 2019, 19, 75-99. [CrossRef]

82. Kumar, S.; Pandey, A.K. Chemistry and biological activities of flavonoids: An overview. Sci. World J. 2013, 2013, 162750. [CrossRef] 
83. Parhiz, H.; Roohbakhsh, A.; Soltani, F.; Rezaee, R.; Iranshahi, M. Antioxidant and anti-inflammatory properties of the citrus flavonoids hesperidin and hesperetin: An updated review of their molecular mechanisms and experimental models. Phytother. Res. 2015, 29, 323-331. [CrossRef]

84. Pietta, P.G. Flavonoids as antioxidants. J. Nat. Prod. 2000, 63, 1035-1042. [CrossRef] [PubMed]

85. Rahman, M.M.; Mostofa, M.G.; Rahman, M.A.; Islam, M.R.; Keya, S.S.; Das, A.K.; Miah, M.G.; Kawser, A.; Ahsan, S.M.; Hashem, A.; et al. Acetic acid: A cost-effective agent for mitigation of seawater-induced salt toxicity in mung bean. Sci. Rep. 2019, 9, 15186. [CrossRef] [PubMed]

86. Xu, D.; Hu, M.J.; Wang, Y.Q.; Cui, Y.L. Antioxidant activities of quercetin and its complexes for medicinal application. Molecules 2019, 24, 1123. [CrossRef] [PubMed]

87. Cianciosi, D.; Forbes-Hernandez, T.Y.; Afrin, S.; Gasparrini, M.; Reboredo-Rodriguez, P.; Manna, P.P.; Zhang, J.; Bravo Lamas, L.; Martinez Florez, S.; Agudo Toyos, P.; et al. Phenolic compounds in honey and their associated health benefits: A review. Molecules 2018, 23, 2322. [CrossRef] [PubMed]

88. Hussain, G.; Zhang, L.; Rasul, A.; Anwar, H.; Sohail, M.U.; Razzaq, A.; Aziz, N.; Shabbir, A.; Ali, M.; Sun, T. Role of plant-derived flavonoids and their mechanism in attenuation of Alzheimer's and Parkinson's diseases: An update of recent data. Molecules 2018, 23, 814. [CrossRef] [PubMed]

89. Kang, H.W.; Lee, S.G.; Otieno, D.; Ha, K. Flavonoids, potential bioactive compounds, and non-shivering thermogenesis. Nutrients 2018, 10, 1168. [CrossRef]

90. Wang, Y.; Chen, S.; Yu, O. Metabolic engineering of flavonoids in plants and microorganisms. Appl. Microbiol. Biotechnol. 2011, 91, 949-956. [CrossRef]

91. Wang, Y.L.; Hou, J.P.; Guo, Y.X.; Ren, J. Antioxidant activities of the extracts of Biebersteinia heterostemon Maxim. Food Ind. 2014, 35, 169-171.

92. Ko, M.J.; Cheigh, C.I.; Chung, M.S. Relationship analysis between flavonoids structure and subcritical water extraction (SWE). Food Chem. 2014, 143, 147-155. [CrossRef]

93. Thavamoney, N.; Sivanadian, L.; Tee, L.H.; Khoo, H.E.; Prasad, K.N.; Kong, K.W. Extraction and recovery of phytochemical components and antioxidative properties in fruit parts of Dacryodes rostrata influenced by different solvents. J. Food. Sci. Technol. 2018, 55, 2523-2532. [CrossRef] [PubMed]

94. Xu, M.; Ran, L.; Chen, N.; Fan, X.; Ren, D.; Yi, L. Polarity-dependent extraction of flavonoids from citrus peel waste using a tailor-made deep eutectic solvent. Food Chem. 2019, 297, 124970. [CrossRef] [PubMed]

95. Guimaraes Drummond, E.S.F.; Miralles, B.; Hernandez-Ledesma, B.; Amigo, L.; Iglesias, A.H.; Reyes Reyes, F.G.; Netto, F.M. Influence of protein-phenolic complex on the antioxidant capacity of flaxseed (Linum usitatissimum L.) products. J. Agric. Food Chem. 2017, 65, 800-809. [CrossRef] [PubMed]

96. Quispe-Fuentes, I.; Vega-Galvez, A.; Aranda, M. Evaluation of phenolic profiles and antioxidant capacity of maqui (Aristotelia chilensis) berries and their relationships to drying methods. J. Sci. Food Agric. 2018, 98, 4168-4176. [CrossRef] [PubMed]

97. Varga, M.; Jojart, R.; Fonad, P.; Mihaly, R.; Palagyi, A. Phenolic composition and antioxidant activity of colored oats. Food Chem. 2018, 268, 153-161. [CrossRef]

98. Zhang, X.X.; Zhang, G.; Jin, M.; Niu, L.X.; Zhang, Y.L. Variation in phenolic content, profile, and antioxidant activity of seeds among different Paeonia ostii cultivated populations in China. Chem. Biodivers. 2018, 15, e1800093. [CrossRef]

99. Soylu, E.M.; Soylu, S.; Kurt, S. Antimicrobial activities of the essential oils of various plants against tomato late blight disease agent Phytophthora infestans. Mycopathologia 2006, 161, 119-128. [CrossRef]

100. Yan, X.; Murphy, B.T.; Hammond, G.B.; Vinson, J.A.; Neto, C.C. Antioxidant activities and antitumor screening of extracts from cranberry fruit (Vaccinium macrocarpon). J. Agric. Food Chem. 2002, 50, 5844-5849. [CrossRef]

101. Dabaghian, F.H.; Entezari, M.; Ghobadi, A.; Hashemi, M. Antimutagenicity and anticancer effects of Biebersteinia multifida DC. Annu. Rev. Res. Biol. 2014, 4, 906-913. [CrossRef]

102. Golshan, A.; Hassanzadeh, S.; Mojdekanloo, M.; Tayarani-Najaran, Z. Effects of Biebersteinia multifida hydro-ethanol extract on proliferation and apoptosis of human prostate cancer and human embryonic kidney cells. Avicenna J. Phytomed. 2016, 6, 671-677. [PubMed]

(C) 2020 by the authors. Licensee MDPI, Basel, Switzerland. This article is an open access article distributed under the terms and conditions of the Creative Commons Attribution (CC BY) license (http://creativecommons.org/licenses/by/4.0/). 